\title{
Potencial terapéutico de los cannabinoides
}

\author{
L. M. Torres ${ }^{1}$, J. M. Trinidad ${ }^{2}$ y E. Calderón ${ }^{1}$

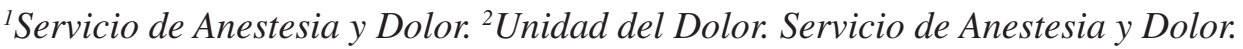 \\ Hospital Universitario Puerta del Mar. Cádiz.
}

Torres LM, Trinidad JM, Calderón E. Potencial terapéutico de los cannabinoides. Rev Soc Esp Dolor 2013; 20(3): 132-136.

\begin{abstract}
Cannabinoids show efficacy in experimental models of acute and chronic pain. They seem safe in trials developed for some indications of pain and others. New technologies have opened new possibilities for treatment by providing new routes of administration. Trials in patients are needed to determine the true role of these substances in the treatment of pain.
\end{abstract}

Key words: Pain. Opioids. Cannabinoid

\section{RESUMEN}

Los cannabinoides demuestran eficacia en modelos experimentales de dolor agudo y crónico. Parecen seguros en los ensayos desarrollados para algunas indicaciones de dolor y otras. Las nuevas tecnologías han abierto nuevas posibilidades de tratamiento al proporcionar nuevas vías de administración. Se precisan ensayos en pacientes para determinar el verdadero rol de estas sustancias en el tratamiento del dolor.

Palabras claves: Dolor. Opioides. Cannabinoides.

Recibido: 12-09-12.

Aceptado: 24-12-12

\section{CAUSAS DEL INADECUADO TRATAMIENTO DEL DOLOR}

El dolor continúa siendo un reto para la medicina actual debido a la ausencia de un adecuado entrenamiento del personal médico. Es preciso reconocer, que durante la formación en la Facultad de Medicina, no se recibe una adecuada enseñanza del tratamiento del dolor, a esto se une el desconocimiento de una estrategia farmacológica adecuada. La simple utilización racional del gran arsenal farmacológico disponible, podría aliviar la mayoría de los cuadros dolorosos. Por otra parte existe una falsa creencia en la poca importancia de los efectos secundarios de los AINE. 10.000 personas se hospitalizan anualmente en España, por complicaciones gastrointestinales relacionadas con AINE. Esto supone un coste aproximado de $30.000 €$ por paciente. Se estima, que en nuestro país, se producen unas 2.000 muertes anuales, relacionadas con la utilización de AINE, por último se demora excesivamente la prescripción de opioides (especialmente los potentes), por la presunta gravedad de sus efectos secundarios mayores (Fig. 1).

\section{ESTADO ACTUAL DE LA UTILIZACIÓN DE LOS ANALGÉSICOS OPIOIDES}

A pesar de que constituyen actualmente el arma más poderosa de que disponemos, para el tratamiento de cualquier cuadro doloroso, ya sea agudo o crónico y de naturaleza oncológica o no oncológica, los opioides son infrautilizados en la clínica práctica. A este respecto, resulta de obligada referencia, la macroencuesta Pain in Europe, realizada entre junio de 2002 y octubre de 2003, en los 15 países más desarrollados de la Unión Europea e Israel (Reino Unido, España, Dinamarca, Irlanda, Francia, Polonia, Holanda, Suiza, Alemania, Suecia, Bélgica, Austria, 
Italia, Noruega, Finlandia e Israel) y que abarcó a 46.000 personas, que fueron entrevistadas telefónicamente, con la finalidad de establecer la prevalencia del dolor crónico no oncológico y su incidencia en la calidad de vida de los pacientes, tratamientos recibidos, grado de satisfacción, etc. (1).

A pesar de establecerse unos criterios de inclusión, sobre lo que es dolor crónico, muy exigentes, que eran:

- Más de 6 meses de evolución.

- Paciente de 18 o más años de edad.

- Haber sufrido dolor durante, el último mes y como mínimo varias veces en semana.

- La intensidad del dolor tiene que ser igual o mayor de 5, en la escala analógica simple.

Los datos que se registraron fueron los siguientes:

- Incidencia:

- Un $19 \%$ de la población europea sufre dolor crónico.

- La mayor incidencia se registra en Noruega, Polonia e Italia (25\%). La menor en España (11 \%).

- $1 / 3$ de los hogares europeos tienen al menos un miembro que padece de dolor crónico.

- Intensidad:

- Se utilizó para la medición de la intensidad del dolor, la escala analógica simple (0-10).

- 2/3 de los pacientes con dolor crónico, sufren un dolor de moderada intensidad.

- El 1/3 restante padecen un dolor severo.

- Evolución:

- Casi la mitad de los pacientes manifiesta padecer un dolor constante. La mayor incidencia de este tipo de dolor se da en Holanda (62\%), seguida de España (61 \%).

- $1 / 3$ de los pacientes sufren de dolor durante las 24 horas del día, 365 días al año.

- Los pacientes llevan con dolor crónico, una media de 7 años. Algunos (21\%) más de 20 años.

- Afectación de la calidad de vida:

- El tratamiento inadecuado del dolor, arruina la vida de los pacientes: afectando a su trabajo, su vida familiar y frecuentemente conduciendo a la depresión (en España, se registra la máxima incidencia de depresión asociada).

- 1/3 de los pacientes manifiestan que están tan afectados por el dolor que "ya no pueden aguantar más".

- 1 de cada 6 pacientes, reconoce haber deseado morir por la intensidad del dolor.

- Acceso a un tratamiento especializado:

- El 70\% es tratado por su médico de Atención Primaria. En menor medida, por traumatólogos (27 $\%)$, neurólogos-neurocirujanos (10\%) y reumató$\log 0 \mathrm{~s}(9 \%)$.

- Solo un $23 \%$ ha sido visto alguna vez por un especialista en tratamiento del dolor.

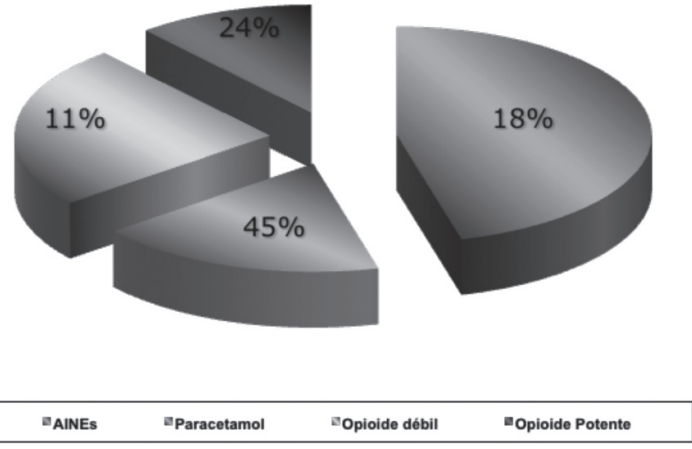

Fig. 1. Fármacos utilizados en el tratamiento del dolor en el estudio Pain in Europe.

- $1 / 3$ de los pacientes piensa que su médico no sabe cómo controlar su dolor.

- 1/4 de los pacientes denuncia que su médico muy rara vez le da importancia a la sintomatología dolorosa, de forma que tienen que exagerar su intensidad, para que les preste atención y les prescriba algún tratamiento.

- El menor uso de opioides potentes se registra en Italia y España. Donde más se usan es en Austria.

- En la mitad de los pacientes se tardó en percibir un alivio adecuado unos 2 años (donde más tiempo en España).

El dramatismo de los datos expuestos, es tan elocuente que habla por sí mismo: es labor de todos los que participamos en el tratamiento del dolor (desde la Medicina Primaria a la Clínica del Dolor), aportar nuestro máximo esfuerzo, para intentar revertir esta intolerable situación, incuestionablemente relacionada con la exigua utilización de los analgésicos más eficaces: los opioides y muy especialmente, de los opioides potentes.

Por si se alberga alguna duda, baste recordar que uno de los indicadores, del grado de calidad de un sistema sanitario, que emplea la OMS, es el consumo de opioides por habitante (Fig. 2) y también aquí, tenemos mucho por conseguir:

\section{LOS CANNABINOIDES. UNA NUEVA ESTRATEGIA PARA EL TRATAMIENTO DEL DOLOR}

Los cannabinoides son los responsables de las propiedades de la marihuana. El término "cannabinoide" engloba muchos más tipos de moléculas que las presentes exclusivamente en la Cannabis sativa (2).

El sistema cannabinoide endógeno (3) constituye un nuevo sistema de comunicación y regulación celular, puede decirse que el sistema endocannabinoide desempeña 


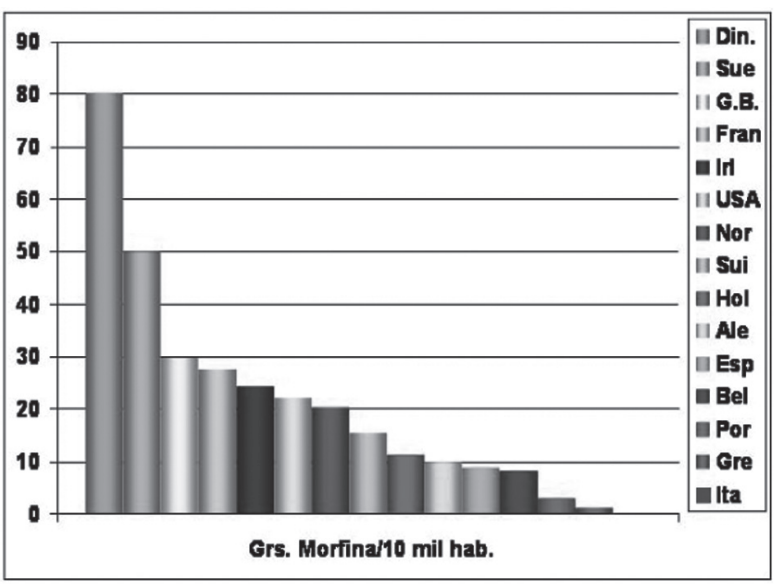

Fig. 2. Consumo de morfina en España.

un papel modulador en diferentes procesos fisiológicos cerebrales $(4,5)$; aunque también en el sistema inmune (6) y cardiovascular (7), y de forma menos clara, a nivel del metabolismo energético y endocrino.

En el cerebro, los endocannabinoides participan en la regulación de la actividad motora del aprendizaje y la memoria (8-10), pero lo que más nos interesan son sobre la nocicepción $(11,12)$.

\section{POTENCIAL TERAPÉUTICO DE LOS CANNABINOIDES}

Seria importante para el uso clínico de estas sustancias el diseño de moléculas más selectivas y con mayor potencia farmacológica que las actualmente en estudio (tetrahidrocannabinol y cannabidiol) (13). Estas nuevas moléculas podrían ser de utilidad en el tratamiento de diversos cuadros dolorosos, ya que sabemos que los cannabinoides son buenos analgésicos, especialmente para el dolor crónico. Esto es consecuencia de la presencia de receptores $\mathrm{CB}_{1}$ en las regiones que participan en el control de la nocicepción, tanto a nivel espinal como a nivel supraespinal $(5,14)$. Existe además una intensa interacción entre la transmisión endocannabinoide y la opiodérgica (15), incluso se han demostrado efectos sinérgicos, lo que ha llevado a sugerir que los cannabinoides podrían ser utilizados para reducir las dosis de morfina en tratamientos de dolor crónico, sin merma del efecto analgésico pero con una notable reducción del potencial adictivo del opiáceo $(3,4,16)$. Asimismo son efectivos en cáncer y sida: gracias a su efecto antiemético y su capacidad de incrementar el apetito, los cannabinoides se están utilizando para reducir las náuseas y el vómito en pacientes con cáncer que son tratados con antineoplásicos, o para reducir la caquexia en pacientes con sida que mantienen de forma crónica tratamientos con compuestos antirretrovirales. Ambos efectos parece que tienen que ver con la activación de receptores $\mathrm{CB}_{1}$ presentes en regiones cerebrales, que participan en el control de la emesis y el apetito (17-19).

\section{APLICACIONES TERAPÉUTICAS DE LOS CANNABINOIDES}

La marihuana (Cannabis sativa L.) se ha empleado en medicina desde hace al menos cincuenta siglos. Sin embargo, la utilización clínica de esta planta y sus componentes activos está hoy en día bastante restringida. En la actualidad se permite en algunos países la prescripción de THC (Marinol@) y/o del cannabinoide sintético nabilona (Cesamet $\left.{ }^{\circledR}\right)$ para estimular el apetito e inhibir las náuseas y el vómito en pacientes de sida o cáncer tratados crónicamente con agentes quimioterapéuticos. De esta forma se puede impedir la pérdida de peso asociada a la quimioterapia, sin duda uno de los efectos más negativos de ella y que conduce a muchos pacientes a abandonarla (19).

Entre otros usos clínicos potenciales de los cannabinoides, cuyo estudio se encuentra en fase III de ensayos clínicos, podríamos destacar el tratamiento del dolor oncológico y neuropático (ver http://www.gwpharm.com; el Sativex®, un medicamento que contiene THC y cannabidiol, se acaba de registrar en Canadá y Reino Unido para el tratamiento del dolor neuropático asociado a la esclerosis múltiple) (17), los trastornos del movimiento (espasmos y temblores) asociados a enfermedades neurodegenerativas como la esclerosis múltiple (ver http://www.cannabis-trial.plymouth.ac.uk), y la recuperación de los traumatismos craneoencefálicos (ver ttp://www.pharmoscorp.com). Existen además otras posibilidades terapéuticas de los cannabinoides que aún se hallan en fases I y/o II de ensayos clínicos o en fases preclínicas. Se sabe que los cannabinoides inhiben el crecimiento de determinadas células cancerosas, lo que podría hacer de ellos agentes antitumorales. $(18,19)$, o el tratamiento del glaucoma (20).

\section{EFECTOS SECUNDARIOS DE LOS CANNABINOIDES EN EL CONTEXTO DE SU APLICACIÓN CLÍNICA}

Los cannabinoides son sustancias bastante seguras. No se han descrito casos de muerte por intoxicación aguda en humanos, y las dosis letales medias son prácticamente imposibles de calcular. La dependencia a cannabinoides observada en algunos modelos animales no parece ser fácilmente extrapolable a humanos que consumen marihuana o sus derivados (21).

Existen asimismo datos que muestran que los cannabinoides no poseen efectos tóxicos generalizados; es más, en 
algunos casos se ha descrito que los cannabinoides podrían proteger a las neuronas frente al daño producido por agentes oxidantes, isquemia y ciertos neurotóxicos.

A pesar de todo ello, el uso de los cannabinoides en medicina está principalmente dificultado por sus efectos psicoactivos, entre los que se incluyen los de tipo afectivo (euforia), somático (somnolencia, descoordinación motora), sensorial (alteraciones en la percepción temporal y espacial, desorientación) y cognitivo (lapsos de memoria, confusión).

Aunque dichos efectos secundarios están dentro de los márgenes aceptados para otros medicamentos, pueden tener en casos concretos una vertiente positiva (relajación, conciliación del sueño, buen humor) y tienden a desaparecer tras su administración prolongada (proceso que se conoce como tolerancia), está claro que al menos para determinados pacientes y patologías sería deseable diseñar cannabinoides que carecieran de acciones psicotrópicas. Puesto que estas dependen de los receptores $\mathrm{CB}_{1}$ del cerebro, la opción más lógica es evitar la activación de estos receptores. Así, se está intentando diseñar (I) compuestos que se unan selectivamente al receptor $\mathrm{CB}_{2}$, (II) compuestos que no atraviesen la barrera hematoencefálica y por tanto no alcancen el cerebro, (III) vías de administración local y controlada fuera del cerebro, y (IV) regímenes de tratamiento (dosis, ciclos) que optimicen los beneficios terapéuticos y minimicen los efectos secundarios. Los próximos años serán sin duda testigos de grandes avances en este terreno.

La comunidad científica se encuentra hoy en día en un punto en el cual se ha acumulado un conocimiento relativamente alto de cómo actúan molecularmente los cannabinoides en el organismo y de cuáles pueden ser algunas de sus aplicaciones terapéuticas más inmediatas. Sin embargo, es necesario llevar a cabo una investigación básica más profunda y ensayos clínicos más exhaustivos. Este artículo pretende ser un reclamo para atraer la atención de investigadores básicos y clínicos a este apasionante tema.

\section{VENTAJAS DE LOS CANNABINOIDES SOBRE LOS OPIOIDES}

- Aceptable eficacia analgésica, además no tienen efecto techo. Sus efectos son dosis-dependientes, a mayor dosificación, mayores efectos analgésicos.

- Pueden asociarse a los AINE, fármacos coadyuvantes (antidepresivos tricíclicos, anticomiciales, paracetamol), y a opioides, para de esta forma conseguir los mejores resultados analgésicos, con la menor incidencia posible, de efectos secundarios. Esto es especialmente importante, en el dolor agudo (dolor posoperatorio), donde el necesario contacto con el opioide, es episódico (uno o varios días), no dando tiempo a que el paciente desarrolle una "tolerancia beneficiosa" a sus efectos secundarios, más comunes (náuseas y vómitos).

- Permiten ser administrados por la mayoría de las vías incluida la inhalatoria. Los cannabinoides demuestran una enorme eficacia en casi todos los cuadros dolorosos en los ensayos con animales. Incluso en aquellos de etiología neuropática, se han realizado algunos estudios que demostrarían la posible utilidad clínica.

- Parecen seguros para el tratamiento del dolor moderado a intenso. Su balance beneficio-riesgo parece interesante.

- Las nuevas tecnologías han abierto nuevas posibilidades de tratamiento. Las formulaciones de liberación sostenida. La paralela evolución de los dispositivos de infusión continua (electrónicos o elastoméricos), mejoraron notablemente el balance beneficio-riesgo, ya que permiten mantenerse unos niveles estables de sus concentraciones plasmáticas y/o intratecales (sin picos ni valles), se obtienen mejores resultados analgésicos y menos efectos secundarios. De esta forma, mejora notablemente el tratamiento domiciliario de muchos pacientes (sobre todo terminales con múltiples síntomas además del dolor). El más reciente desarrollo de los parches transcutáneos, ha supuesto un nuevo empuje, al tratamiento adecuado del dolor crónico, si los cannabinoides pudieran administrarse por esta vía seria una gran ventaja para su uso.

CORRESPONDENCIA:

LM Torres

1mtorres@arrakis.es

\section{BIBLIOGRAFÍA}

1. Breivik H, Collett B, Ventafridda V, et al. Survey of chronic pain in Europe: Prevalence, impact on daily life, and treatment. EJP. 2006;10:287-333.

2. Mackie K. Cannabinoid receptors as therapeutic targets. Annual Review of Pharmacology and Toxicology. 2005; 46:101-22.

3. De Vries TJ, Shaham Y, Homberg JR, et al. A cannabinoid mechanism in relapse to cocaine seeking. Nature Med. 2001; 7:1151-4.

4. Fattore L, Martellota MC, Cossu G, et al. CB1 cannabinoid receptor agonist WIN55, 212-2 decreases intravenous cocaine self-administration in rats. Behav Brain Res. 1999;104:141-8.

5. Fernández-Ruiz J, Lastres-Becker I, Cabranes A, et al. Endocannabinoids and basal ganglia functionality. Protag Leukot Essent Fatty Acids. 2002;66:257-67.

6. Parolaro D. Presence and functional regulation of cannabinoid receptors in immune cells. Life Sci. 1999;65:637-44.

7. Hillard CJ. Endocannabinoids and vascular function. J Pharm Exp Ther. 2000;294:27-32.

8. Hampson RE, Deadwyler SA. Cannabinoids, hippocampal function and memory. Life Sci. 1999;65:715-23. 
9. Fernández Ruiz JJ, Berrendero F, Hernández ML, et al. The endogenous cannabinoid system and brain development. Trends Neurosci. 2000;23:14-20.

10. Piomelli D. The molecular logic of endocannabinoid signalling. Nature Reviews Neuroscience. 2003;4:873-84.

11. Di Marzo V, Melck D, Bisogno T, et al. Endocannabinoids: endogenous cannabinoid receptor ligands with neuromodulatory action. Trends Neurosci. 1998;21:521-8.

12. Walker JM, Hohmann AG, Martin WJ, et al. The neurobiology of cannabinoid analgesia. Life Sci. 1999;65:66573.

13. Piomelli D, Giuffrida A, Calignano A, et al. The endocannabinoid system as a target for therapeutic drugs. Trends Pharmacol Sci. 2000;21:218-24.

14. Romero J, Lastres Becker I, de Miguel R, et al. The endogenous cannabinoid system and the basal ganglia: biochemical, pharmacological and therapeutic aspects. Pharm Ther. 2002;95:137-52.
15. Maldonado, R.; Valverde, O., Berrendero, F., Involvement of the endocannabinoid system in drug addiction. TRENDS in Neuroscionces 2006;29, 225-232.

16. Manzanares J, Corchero J, Romero J, et al. Pharmacological and biochemical interactions between opioid and cannabinoids. Trends Pharmacol Sci. 1999;20:287-94.

17 Pertwee RG. Cannabinoids and multiple sclerosis. Pharmacol Ther. 2002;95:165-74.

18. Guzmán M. Cannabinoids: potential anticancer agents. Nature Reviews Cancer. 2003;3:745-55.

19. Hall W, Christie M, Currow D. Cannabinoids and cancer: causation, remediation, and palliation. The Lancet Oncology. 2005;6:35-42.

20. Jarvinen T, Pate DW, Laine K. Cannabinoids in the treatment of glaucoma. Pharmacol Ther. 2002;95:203-20.

21. Hine B, Friedman E, Torrelio M, et al. Morphine dependent rats: blockade of precipitated abstinence by tetrahydrocannabinol. Science. 1975;187:443-5. 